\title{
Estimación automática del gasto energético de la actividad física de niños en videojuegos de ejercicio con el sensor Kinect
}

\author{
Benjamín Gutiérrez-Serafín ${ }^{1}$, Humberto Pérez-Espinosa ${ }^{1,2}$, \\ Ismael Edrein Espinosa-Curiel ${ }^{1}$, Pedro N. Figueroa-García ${ }^{3}$, \\ Edgar E. Pozas-Bogarín ${ }^{1}$, Juan Martínez-Miranda ${ }^{1,2}$ \\ 1 CICESE-UT3, Tepic, Nayarit, México \\ 2 Catedrático CONACyT \\ 3 Universidad Autónoma de Nayarit, Tepic, Nayarit, México \\ begutierrezse@outlook.com, hperez@cicese.mx, ecuriel@cicese.mx
}

\begin{abstract}
Resumen. En este artículo se presenta EGEKN, el cual es un método para la estimación automática del gasto energético dirigido a niños de entre 7 y 10 años de edad durante sesiones de juego con videojuegos activos. Este método toma como entrada los datos de seguimiento de las juntas del modelo esquelético capturados con un sensor Kinect para calcular el trabajo mecánico del cuerpo del jugador. Adicionalmente, este método considera información contextual como la edad, el sexo, el peso y el índice de masa corporal de los jugadores, el tipo de actividad realizada, el porcentaje de grasa corporal y la frecuencia cardíaca media. A partir de la representación de esta información en vectores de características se entrenó un modelo de regresión para la estimación del gasto energético. Para desarrollar y validar este método se capturaron datos de 46 niños de entre 7 y 10 años de edad. Los participantes jugaron 12 videojuegos activos con diferentes tipos de movimiento e intensidades de actividad física. El modelo entrenado obtuvo una correlación de 0.85 , lo cual es comparable con la precisión de métodos similares encontrados en la literatura, pero dirigidos a adultos.
\end{abstract}

Palabras clave: aprendizaje máquina, estimación de calorías, caracterización de movimientos.

\section{Automatic Estimation of the Energy Expenditure of Children Physical Activity in Exergames Using the Kinect Sensor}

Abstract. In this article, we present a method for automatic estimation of children energy expenditure during playing sessions with exergames, 
called EGEKN. This method calculates the mechanical work of the player's body using the tracking data of the joints of the skeletal model captured with a Kinect sensor. It also uses contextual information such as the age, sex, weight, and body mass index of the players, the type of activity performed, the percentage of body fat and the average heart rate. Using feature vectors to represent this information, we trained a regression model to estimate the energy expenditure. We collected data from 46 children aged between 7 and 10 years old to develop and validate this method. The participants played with12 exergames with different types of movement and physical activity intensities. The trained model obtained a correlation of 0.85 , which is comparable with the accuracy of similar methods found in the literature but aimed at adults.

Keywords: machine learning, energy expenditure estimation, body movements characterization.

\section{Introducción}

La obesidad infantil aumentó dramáticamente durante las últimas décadas a nivel mundial, alcanzando niveles epidémicos [4,23]. La obesidad infantil está asociada a la inactividad física y un estilo de vida sedentario de los niños. Debido a lo anterior, una de las principales estrategias para reducir la obesidad infantil es aumentar la actividad física y reducir las conductas sedentarias de los niños [17]. Gracias al desarrollo de nuevos y novedosos dispositivos de interacción Humano-Computadora (ej. Controles Wii, Kinect, y cámaras de profundidad), los videojuegos activos (exergames) surgieron como una estrategia emergente para promover la actividad física en niños. Este tipo de videojuegos proveen métodos emocionantes, innovadores y atractivos para atraer la atención, educar y promover cambios en las intenciones y los comportamientos humanos $[1,14]$.

Estudios recientes revelan que la mayoría de los videojuegos activos producen un nivel medio de intensidad de ejercicio; y muy pocos producen un nivel intenso de ejercicio, el cual es requerido para obtener beneficios cardiovasculares considerables [15]. Para tener un mayor impacto en la salud, los videojuegos activos podrían ajustar automáticamente la intensidad de la actividad física de acuerdo al perfil y desempeño del jugador; sin embargo, uno de los retos más importantes para lograr tal fin es medir efectivamente el gasto energético (GE) de los jugadores. El uso de los métodos actuales para medir el GE en los videojuegos activos se ve afectado por su precisión, complejidad de uso, y sus costos. Por ejemplo, la precisión de los métodos que utilizan acelerómetros, unos de los mas ampliamente utilizados, depende del número de dispositivos utilizados, requiriendo en ocasiones hasta 5 dispositivos [12]. Por su parte, los sistemas de análisis metabólico de gases son uno de los mas precisos; sin embargo, son caros y requieren un esfuerzo significativo para ponerlo a punto. Una limitación de la mayoría de los métodos de GE es que son invasivos debido a que requieren que los jugadores porten sensores de medición durante toda la actividad física. Como una alternativa a estos métodos, recientemente se han desarrollado métodos no 
invasivos de estimación del GE que utilizan el sensor Microsoft Kinect para rastrear los movimientos corporales sin la necesidad de portar ningún instrumento. Sin embargo, estos métodos han sido desarrollados para adultos y, hasta donde tenemos conocimiento, no existe un método específico para niños en edad escolar. Debido a lo anterior, en este trabajo se presenta EGEKN, el cual es un método no invasivo para la estimación automática del gasto energético dirigido a niños de entre 7 y 10 años de edad durante sesiones de juego con videojuegos activos. EGEKN aprovecha las capacidades del sensor Microsoft Kinect para rastrear de forma precisa los movimientos de las articulaciones esqueléticas del jugador, y con base en esa información estimar el gasto energético. EGEKN puede ser integrado a videojuegos activos para mostrar el gasto energético de los jugadores, o para ajustar la intensidad de actividad física en tiempo real y de forma personalizada.

\section{Trabajo previo}

A continuación se presentan investigaciones que abordan la estimación del gasto energético empleando el sensor Kinect como medio de captura de movimiento. La herramienta denominada ViziCal desarrollada en [9] es un enfoque basado en regresión que mapea datos cinemáticos recopilados por el sensor Kinect y reporta el gasto de energía en unidades metabólicas de reposo (MET). Este sistema toma en consideración la información de la ubicación y la aceleración de las articulaciones esqueléticas para predecir la actividad física en videojuegos activos. Los datos se recopilaron de un grupo de 9 participantes masculinos entre 18 y 25 años que se ejercitaban regularmente. Para desarrollar el algoritmo de estimación se usaron, máquinas de soporte vectorial para tres conjuntos diferentes de características: aceleración cinemática, posición de articulaciones y la combinación de estos dos. Se empleo un enfoque de validación cruzada dejando uno fuera. ViziCal resultó ser más preciso que las técnicas convencionales basadas en acelerómetros. Otra contribución es la desarrollada en [13] donde se propone un método que calcula el consumo de energía en tiempo real al evaluar la suma de la energía requerida para superar la energía potencial gravitatoria de cada parte del cuerpo del modelo esquelético. En este experimento se utilizó un conjunto de vídeos de baile para obtener y modelar un cuerpo humano, el cual consta de 10 partes formadas por 15 nodos de articulaciones. Al monitorear el cambio de posición de cada parte del cuerpo, se puede conocer la energía empleada para cada uno de ellos y la sumatoria de sus energías da origen al consumo total de energía del cuerpo completo. Una limitación es que el consumo de calorías se estimó en función de un avatar y no directamente del cuerpo de los participantes.

Por otro lado, el estudio [21] propone un método similar para el consumo de calorías en rutinas de aerobics. En esta investigación se usó el sensor Kinect para monitorizar 10 juntas del modelo esquelético, las cuales fueron consideradas como un acelerómetro de 3 ejes. Además, se contempló el ritmo cardíaco para complementar la evaluación de múltiples modelos de regresión. Este método obtuvo mejores resultados en comparación a los obtenidos con un solo acelerómetro y 
sin requerir numerosos entrenamientos para crear un modelo estable. En [16] se propuso un modelo más robusto desde un enfoque de aprendizaje estadístico multivariado. Este modelo es capaz de estimar el gasto energético con base en el trabajo mecánico interno y externo realizado en 4 ejercicios individuales diferentes. Con el sensor Kinect, se localizó en un sistema local de coordenadas, 20 juntas del modelo esquelético para rastrear en cada momento del ejercicio los movimientos realizados por los participantes. Posteriormente se calcula el trabajo mecánico realizado en el cuerpo tomando en cuenta los cambios de su energía mecánica y finalmente se estima el gasto energético.

En general, los métodos de estimación del gasto energético que usan el sensor Kinect para medir el movimiento han obtenido buenos resultados y se han adaptado a diferentes actividades físicas. Sin embargo, hasta donde tenemos conocimiento no se ha publicado ningún trabajo que reporte una técnica de estimación de gasto energético en sesiones de juego activo específica para niños como sujeto de prueba. Por tal motivo, la presente investigación se centra en desarrollar una técnica específica para niños que estime el gasto energético durante actividad física con videojuegos activos.

\section{Recolección de datos}

Para desarrollar el método de estimación se recolectaron datos de 46 estudiantes (23 niños y 23 niñas) de entre 7 y 10 años, inscritos en una escuela primaria pública. El estudio se desarrolló durante tres meses. Previo al inicio de la recolección de los datos, se revisó el protocolo del experimento con un experto en educación física infantil con el objetivo de afinar los ejercicios y su intensidad para evitar poner en riesgo la salud y bienestar de los participantes. Posteriormente, se obtuvo la autorización del director de la escuela y los maestros para realizar el estudio. Finalmente, se obtuvo el consentimiento informado firmado por los padres y el consentimiento verbal de parte de los niños. Las características de los participantes se muestran en la Tabla 1.

Tabla 1. Características de los participantes.

\begin{tabular}{ccccc}
\hline Características & Promedio & Desviación estandar & Máximo & Mínimo \\
\hline Masa (kg) & 41.3 & 9.27 & 67.4 & 25.2 \\
Altura (cm) & 138 & 8.51 & 153 & 121 \\
Edad & 8.9 & 0.86 & 10 & 7 \\
IMC & 21.3 & 2.82 & 29.5 & 16.6 \\
BFP & 29.1 & 6.05 & 46.3 & 16.2 \\
\hline
\end{tabular}

Los niños participaron en binas en sesiones de juego que duraron en promedio 90 minutos, de los cuales solo 26 minutos fueron de actividad física. Los niños podían terminar su participación en el momento que ellos quisieras. Los niños 
pasaban en binas con el objetivo de alternar sus tiempos de ejercicio y descanso además de que reducía el tiempo requerido para dar las instrucciones a los jugadores. A los participantes se les recomendó asistir con ropa cómoda para realizar adecuadamente las actividades. Cada sesión de juego se dividió en las siguientes etapas:

1. Se les colocaba a los participantes un sensor de pulso cardíaco Polar OH1 conectado a la aplicación Polar Beat, con el objetivo de capturar la frecuencia cardíaca y el gasto energético. Además, se calibraba el sensor Microsoft Kinect para capturar adecuadamente el movimiento de sus cuerpos.

2. A los participantes se les explicaba cómo jugar, el objetivo de los videojuegos, y como acumular el mayor puntaje en el tiempo establecido haciendo correctamente lo que los videojuegos requerían.

3. Los participantes realizaban una sesión de calentamiento dinámico de entre 8 a 10 minutos que incluía movimientos articulares: flexiones, extensiones, abducciones y aducciones de hombros caderas, rodillas y tobillos. Para definir las actividades de calentamiento se siguieron las recomendaciones de [5].

4. Los participantes jugaban 3 sesiones de juego de 5 minutos cada una con un videojuego activo de acción. Había cinco posibles videojuegos activos de acción: FoodRateMaster (c), FatCombat (c), DishMaster (c), SuperMarketRace (C), y FoodHitTravel (c). Todos los videojuegos son desarrollos propios y tienen en tramite el registro de propiedad intelectual. Cada videojuego requería un conjunto de movimientos corporales para poder jugar (ej. desplazamientos laterales, saltos, agacharse, tocar objetos con las manos, etc.). Se empezaba con una intensidad baja y en las dos sesiones subsecuentes se le aumentaba la intensidad sin superar las 197 pulsaciones por minuto (PPM) el cual es el limite máximo para niños y adolescentes [7]. Las intensidades son: baja (140 PPM), media (160 PPM) y alta $(>160<180$ PPM). Después de jugar cada sesión de juego, el participante tenía 5 minutos de reposo absoluto y el otro miembro de la bina iniciaba a jugar lo mismo que el jugador que estaba descansando.

5. Los participantes jugaban 3 videojuegos activos de reto que requerían realizar ejercicios de alta intensidad (salto de mariposa, skipping y taloneo). La duración de cada videojuego era de 40 segundos y después tenían 40 segundos de recuperación pasiva. Durante el reposo el otro miembro de la bina empezaba a jugar.

6. Los participantes jugaban 6 videojuegos activos de reto que requerían realizar ejercicios de fuerza muscular (bíceps, desplante frontal, desplante lateral, elevación de hombros frontal, elevación de hombros lateral y sentadillas) durante 90 segundos con el mismo tiempo de recuperación pasiva. Durante el reposo el otro miembro de la bina empezaba a jugar.

7. Los participantes realizaban estiramiento para volver a la calma.

Se recopilaron datos del movimiento del cuerpo, frecuencia cardíaca y gasto energético. Se utilizó la aplicación Polar Beat para el registro del gasto energético. Para el caso de los videojuegos de 5 minutos se tomó cada minuto, y en los 
videojuegos de ejercicio de alta intensidad y fuerza muscular, una sola vez al finalizar el juego. Durante el estudio se obtuvieron un total de 521 capturas de movimiento.

\section{Metodología}

Con ayuda del dispositivo Kinect se capturaron las ubicaciones de las juntas del esqueleto de los usuarios en un sistema de referencia local de coordenadas, a fin de localizarlos en cualquier tiempo de la sesión de juego y con ellos determinar variables esenciales para el análisis del movimiento del cuerpo humano. Una vez obtenidos estos parámetros básicos, se implementó el enfoque propuesto por Nathan [16] para el cálculo del trabajo externo e interno, y subsecuentemente, estimar el gasto energético empleando aprendizaje máquina para modelar estas características junto con otras propuestas por los autores del presente trabajo.

\subsection{Descripción general del método propuesto}

El objetivo principal de este trabajo de investigación es proponer un enfoque más amplio al reportado en [16] al implementar un algoritmo que estime el gasto energético durante actividades físicas realizadas en sesiones de juego serio orientados a disminuir el sobrepeso en niños y adaptar el modelo a esta población. Por este motivo, se propuso evaluar diferentes algoritmos de aprendizaje con 3 conjuntos de características diferentes para examinar y comparar su eficacia cuando esta técnica se aplica a actividades físicas más complejas que las estudiadas por Nathan (sentadilla, salto de mariposa, movimiento de brazos y salto de pie). Las funciones de cada una de las fases principales de nuestro método se describen en las siguientes subsecciones.

\subsection{Captura de coordenadas de las juntas}

Se utilizó el sensor Microsoft Kinect V2 y su SDK para la obtener las coordenadas de 20 juntas. El modelo del dispositivo fue lanzado en el año 2014 y discontinuado en el 2017. No obstante, Microsoft tiene planes de un nuevo lanzamiento con un módulo de cámara más sofisticado y tecnología de inteligencia artificial basada en la nube de Microsoft [2]. 19 juntas representan una articulación del cuerpo y una representa el centro del modelo esquelético del cuerpo. Está información es representada en un vector XYZ de valores decimales en metros con respecto a la posición del Kinect. La estructura con la que trabajan las juntas sigue un modelo de árbol jerárquico donde se parte de una junta raíz y a partir de ella se van relacionando las demás juntas. Se capturaron fotogramas de las 20 juntas cada 30 milisegundos y se almacenó en un archivo CSV junto con información del participante. 
Estimación automática del gasto energético de la actividad física de niños en videojuegos...

\subsection{Cálculo de propiedades de los segmentos del cuerpo}

De manera general, la obtención del centro de masa del cuerpo completo, así como otras propiedades inerciales es una tarea indispensable cuando se necesita realizar un análisis cuantitativo del movimiento [20]. Dado que el cuerpo humano es un sistema formado por segmentos conectados entre sí, la distribución de masa del cuerpo varia continuamente dependiendo a la posición de sus segmentos, lo que ocasiona que el centro de gravedad cambie de lugar constantemente. Esta dependencia continua de la postura corporal provoca que el hallazgo del centro de masa del cuerpo completo sea más complejo en comparación con el de simples cuerpos rígidos. Por consiguiente, diversos métodos para encontrar su ubicación se han ideado. La opción más conveniente dadas las características del proyecto, es la determinación del centro de gravedad del cuerpo completo por medio de la suma ponderada de todos los centros de masas individuales de los segmentos. Para esta tarea, se recurrió a los parámetros de segmentos del cuerpo proporcionados por De Leva [3] para primeramente calcular los centros de masa de los segmentos individuales con las siguientes ecuaciones para cada uno de sus componentes cartesianos para todos los fotogramas obtenidos en cada sesión de juego:

$$
\begin{aligned}
x_{\text {seg }} & =x_{p}+R_{p}\left(x_{d}-x_{p}\right), \\
y_{\text {seg }} & =y_{p}+R_{p}\left(y_{d}-y_{p}\right), \\
z_{\text {seg }} & =z_{p}+R_{p}\left(z_{d}-z_{p}\right) .
\end{aligned}
$$

Es decir, la posición del centro de masa del segmento $\left(x_{s e g}, y_{s e g}, z_{\text {seg }}\right)$ se puede determinar a partir de las coordenadas del punto proximal $\left(x_{p}, y_{p}, z_{p}\right)$ y distal $\left(x_{d}, y_{d}, z_{p}\right)$ de la parte del cuerpo a analizar y el porcentaje de su longitud $R_{p}$. Posteriormente, el centro de masa del cuerpo completo $\left(x_{c c}, y_{c c}, z_{c c}\right)$ es obtenido empleando la fórmula 2 para ubicar sus coordenadas en el espacio, donde $P_{i}$ y $\left(x_{i}, y_{i}, z_{i}\right)$, son respectivamente, la proporción de masa y cada uno de los componentes rectangulares del centro de masa del segmento i.

$$
x_{c c}=\frac{\sum\left(P_{i} x_{i}\right)}{\sum P_{i}}, y_{c c}=\frac{\sum\left(P_{i} y_{i}\right)}{\sum P_{i}}, z_{c c}=\frac{\sum\left(P_{i} z_{i}\right)}{\sum P_{i}} .
$$

\subsection{Cálculo de la energía y trabajo mecánico}

Para llevar a cabo la caracterización del modelo de estimación del gasto energético, se consideró el trabajo mecánico interno y externo realizado en el sistema por variaciones en su energía mecánica. Como se describe en [22], es uno de los métodos más destacados puesto que provee información importante no solo de la mecánica del cuerpo, sino también de su movimiento. Tal como se mencionó con anterioridad, se adoptó el método propuesto por Nathan D. [16] el cual se divide en dos partes. La primera fase se encarga de analizar las interacciones de fuerzas externas capaces de causar cambios de movimiento en el centro de masa de todo el cuerpo relativo al entorno, lo que origina alteraciones en la energía mecánica del sistema, produciendo lo que se conoce como trabajo externo. La 
energía en cualquier instante asociada con el trabajo externo del centro de masa del cuerpo completo puede ser obtenida con la siguiente ecuación:

$$
E_{C O M}(t)=M g H(t)+\frac{1}{2} M V_{c g}(t)^{2}
$$

donde $M$ es la masa total de todo el cuerpo, $g$ la gravedad, $H$ la altura del centro de masa y $V_{c g}$ la velocidad lineal del centro de masa relativo al entorno. Una vez que calculada la energía en cualquier tiempo t, se prosigue con el cálculo del trabajo externo que se define de la siguiente manera:

$$
W_{\text {ext }}(t)=E_{C O M}(t+1)-E_{C O M}(t)
$$

En cuanto a la segunda fase, se evalúa los cambios de energía ocasionados por fuerzas internas del sistema y la transferencia de energía interna que existe entre un segmento y segmentos adyacentes dentro de la misma extremidad. Originando de esta manera el trabajo provocado por la diferencia de energía mecánica relativa al centro de masa del cuerpo completo, el cual se denomina trabajo interno. La energía en cualquier momento $t$ asociada con el trabajo interno del segmento $i$ se expresa como:

$$
E_{\text {int }, i}(t)=\frac{1}{2} m_{i} V_{r, i}(t)^{2}+\frac{1}{2} m_{i} K_{i}^{2} w_{i}(t)^{2}
$$

donde $m_{i}$ es la masa del segmento, $V_{r i}$ es la velocidad relativa del centro de masa del segmento $i$ con respecto al centro de masa del cuerpo completo, $K_{i}$ es el radio de giro del centro de masa y $w_{i}$ es la velocidad angular del segmento $i$. De manera similar que el paso anterior, el trabajo interno del segmento $i$ está dado por la ecuación:

$$
W_{\text {int }, t}(t)=E_{\text {int }, i}(t+1)-E_{\text {int }, i}(t)
$$

Teniendo en cuenta que el trabajo realizado por los músculos varía dependiendo del tipo de contracción, también se tomó en consideración diferenciar entre las dos clases que se pueden presentar. Knuttgen y Kraemer [10] exponen que, en caso de que la fuerza producida sea lo suficientemente fuerte para superar la resistencia, el musculo se acorta a medida que se contrae de manera concéntrica (trabajo positivo). En cambio, cuando la contracción es excéntrica (trabajo negativo), la fuerza generada no es suficiente para sobrepasar la carga, lo cual causa que el músculo se alargue mientras se contrae de manera excéntrica. A la luz de este relevante hecho, la suma de todo trabajo positivo efectuado durante la actividad física fue almacenada en las variables de trabajo interno positivo y trabajo externo positivo. De igual forma, la sumatoria de todo trabajo negativo realizado durante la sesión de juego se administró en las variables de trabajo interno negativo y trabajo externo negativo. Cabe señalar que, con respecto a las variables de trabajo interno, se decidió separarlo en extremidades inferiores y superiores. Finalmente, después de caracterizar el movimiento de esta manera se obtuvieron 6 características relevantes (ver Tabla 2). 
Estimación automática del gasto energético de la actividad física de niños en videojuegos...

\subsection{Obtención de características contextuales}

Tal como se presentó en [8] variables como el ritmo cardíaco, edad, genero, entre otros, contribuyeron de manera significativa para que su modelo de regresión reportara un coeficiente de correlación altamente positivo al momento de estimar el gasto energético durante sus experimentaciones. Por lo tanto, en este trabajo se propone la inclusión de los datos proporcionados por el perfil del jugador que informan sobre las características de los participantes para mejorar la efectividad del modelo. Además de la edad y el género, la funcionalidad especial incluida en nuestra plataforma mantiene registros del tamaño del cuerpo y algunas composiciones corporales de los usuarios que se ingresan al sistema cuando los niños se registran al comienzo de su primera sesión. En adición a estas características personales, también se decidió agregar información sobre la sesión jugada, es decir, descripción acerca del nivel de intensidad, tipo de actividad física o videojuego seleccionado. Por último, el dispositivo empleado para el monitoreo de las calorías quemadas por sesión, Polar OH1, incluye la utilidad de poder llevar un seguimiento de las pulsaciones por minuto presentes durante la sesión de juego. Por consiguiente, se optó por incorporar el ritmo cardíaco como una característica adicional a nuestro modelo de estimación de gasto energético.

Representación del vector características. Esta sección proporciona una perspectiva general de la caracterización empleada para el sistema de estimación de gasto energético. Una vez capturados y calculados los atributos planteados en las secciones anteriores se prosiguió con la formación del vector característica para su posterior análisis.

Las primeras 6 características adquiridas durante el procesamiento de los datos suministrados por el dispositivo Kinect están vinculadas con el trabajo mecánico medido en kilojulios e interpretado como variable cuantitativa el cual es realizado durante la actividad física. El trabajo mecánico se representa como la sumatoria a través del tiempo tanto del trabajo positivo y negativo empleado para mover el centro de masa del cuerpo completo por el entorno, como del trabajo positivo y negativo provocados por cambios en la energía mecánica relativa al centro de masa del cuerpo completo para mover sus elementos superiores e inferiores.

En cuanto a los datos provistos por el sistema, se encuentran asociados en mayor medida a información recabada para el perfil de jugador, el cual consta de la edad del participante, genero, peso, altura, porcentaje de grasa e índice de masa corporal, donde las primeras dos variables fueron contempladas como valores discretos y las cuatro restantes como valores continuos. Asimismo, la plataforma de videojuegos brindó información sobre el tipo de actividad y la sesión seleccionada. Para representar esta información se aplicó la técnica de codificación one-hot, que consiste en remplazar la variable categórica por una nueva variable binaria por cada clase contenida originalmente. Finalmente, el dispositivo de recopilación de gasto energético provee adicionalmente la frecuencia cardíaca media como características de tipo continuo. Este último atributo 
y las calorías quemadas por sesión se encuentran a nuestra disposición una vez que se haya concluido la actividad física. La Tabla 2 lista las características analizadas en este trabajo.

Tabla 2. Lista de atributos utilizados para el análisis.

Trabajo mecánico

\begin{tabular}{l} 
Trabajo externo positivo \\
Trabajo externo negativo \\
Trabajo interno positivo superior \\
Trabajo interno negativo superior \\
Trabajo interno positivo inferior \\
Trabajo interno negativo inferior \\
\hline Datos del participante \\
\hline Edad \\
Genero \\
Peso \\
Índice de masa corporal \\
\hline Información de actividad \\
\hline Tipo de actividad \\
Sesión seleccionada \\
Porcentaje de grasa corporal \\
\hline Dispositivo de medición \\
\hline Frecuencia cardíaca media \\
\hline
\end{tabular}

\subsection{Estimación del gasto energético}

En este estudio se utilizaron diferentes conjuntos de características y se aplicaron varias técnicas de aprendizaje máquina con el objetivo de identificar la combinación más precisa para la estimación del gasto energético. Asimismo, se consideró evaluar cada una de los tipos de sesión tanto de manera independiente como en conjunto. Dicho de otra forma, se agruparon las capturas por tipo de sesión y se realizaron experimentaciones para sesiones de juego, videojuegos de reto y la mezcla de estos tipos. Para evaluar el método propuesto, se decidió entrenar los modelos utilizando solo las 6 características obtenidas por el proceso del cálculo del trabajo mecánico en el primer experimento. En la siguiente etapa de experimentación, se incorporaron las características proporcionadas por el perfil de jugador, pero excluyendo el ritmo cardíaco promedio puesto que esta variable es obtenible una vez que se termina la sesión. Se procuró observar el desempeño del modelo descartando esta variable para visualizar su rendimiento en caso de querer implementar el algoritmo en tiempo real. De igual manera, se examinó el comportamiento de los modelos de regresión al agregar el ritmo cardíaco al vector característica. Como última tarea, se crearon modelos de entrenamiento y prueba independientes del sujeto con el fin de no incluir muestras de un mismo niño en los conjuntos de entrenamiento y de 
Estimación automática del gasto energético de la actividad física de niños en videojuegos...

prueba y así evitar cualquier tipo de dependencia de los modelos entrenados con propiedades de los individuos. En cuanto a los algoritmos de regresión empleados, se propuso explorar el rendimiento de random forest [11], proceso gaussiano [18], perceptrón multicapa [6] y máquinas de soporte vectorial [19] para todas las experimentaciones de este estudio y mostrar sus rendimiento en las tablas 3, 4, 5 y 6 empleando como métricas de desempeño el coeficiente de correlación de Pearson, error medio absoluto y el error cuadrático medio. Además, se validó la estabilidad de estos algoritmos aplicando dos métodos de validación. 1) Validación cruzada de 10 iteraciones: En esta técnica, un $90 \%$ de las muestras del conjunto de datos es entrenado para crear un modelo que es probado con el $10 \%$ restante de los datos. Este proceso es repetido 10 veces usando diferentes conjuntos de entrenamiento/prueba para cada ocasión. 2) Validación 80/20: Para este esquema de validación, se crea un modelo de entrenamiento con el $80 \%$ de las muestras del conjunto total de datos. El $20 \%$ restante es utilizado para probar el modelo.

\section{Resultados}

Como puede observarse en la Tabla 3 , las mejores métricas de regresión tanto en validación cruzada de 10 pliegues como en $80 \%$ entrenamiento / $20 \%$ prueba fueron obtenidos cuando los conjuntos de entrenamiento y prueba contenían muestras de los dos tipos de videojuegos (acción y reto) que se probaron. No obstante, cuando se analizaron por separado los dos tipos de juegos se redujeron de manera significativa los coeficientes de correlación y aumentaron las métricas del error, especialmente en las sesiones de juego de acción.

De acuerdo con las suposiciones establecidas, el comportamiento del modelo mejoró en la segunda experimentación, puesto que las características incluidas para esta etapa lograron que se obtuvieran resultados satisfactorios, no solo para el conjunto mezclado, sino también para el grupo de entrenamiento formado solo por videojuegos de reto. No se observó mejora significativa para el modelo constituido por sesiones de juego de acción, ver Tabla 4. Por otro lado, cuando se realizó experimentación tomando en consideración el ritmo cardíaco, se obtuvieron resultados más precisos que en los dos experimentos anteriores. Vale la pena señalar que, en esta ocasión, el modelo conformado por sesiones de juego de acción presentó un aumentó en los coeficientes de correlación, especialmente cuando se implementó el algoritmo perceptrón multicapa. Los resultados obtenidos de estos dos experimentos se encuentran localizados en la Tabla 5.

Para la última fase de experimentación, se limitó a estudiar solo las características utilizadas en la segunda etapa para visualizar el rendimiento que se originaría en un entorno real de uso. Los resultados obtenidos en este último experimento pueden visualizarse en la Tabla 6. Como se puede apreciar, la eficacia de los modelos se redujo si se comparan con los 2 experimentos previos, especialmente el de sesiones de juego, ya que no se fue capaz de obtener valores favorables para ninguno de los algoritmos de regresión empleados. Sin embargo, 
Benjamín Gutiérrez-Serafín, Humberto Pérez-Espinosa, Ismael Edrein Espinosa-Curiel, et al.

Tabla 3. Resultados de la primera experimentación utilizando solo las 6 características del trabajo mecánico.

\begin{tabular}{|c|c|c|c|c|c|c|c|c|}
\hline \multicolumn{9}{|c|}{ Todos } \\
\hline \multirow{2}{*}{ Resumen } & \multicolumn{4}{|c|}{$80 / 20$} & \multicolumn{4}{|c|}{$10 \mathrm{FCV}$} \\
\hline & $\mathrm{RF}$ & GP & MLP & SVM & $\mathrm{RF}$ & GP & MLP & SVM \\
\hline Correlación & 0.78 & 0.66 & 0.73 & 0.75 & 0.79 & 0.73 & 0.72 & 0.77 \\
\hline MAE & 6.63 & 11.44 & 9.35 & 7.25 & 0.58 & 11.91 & 7.77 & 6.17 \\
\hline RMSE & 12.18 & 15.21 & 15.17 & 13.21 & 11.12 & 14.63 & 12.70 & 11.57 \\
\hline
\end{tabular}

\begin{tabular}{|cccc|ccccc|}
\hline \multicolumn{8}{c|}{ Videojuego de reto } \\
\multirow{2}{*}{ Resumen } & \multicolumn{4}{c|}{$80 / 20$} & \multicolumn{4}{c|}{ 10FCV } \\
& RF & GP & MLP & SVM & RF & GP & MLP & SVM \\
\hline Correlación & 0.55 & 0.41 & 0.54 & 0.43 & 0.46 & 0.29 & 0.40 & 0.35 \\
MAE & 2.10 & 2.53 & 2.20 & 2.41 & 2.03 & 2.3 & 2.39 & 2.16 \\
RMSE & 2.89 & 3.32 & 2.98 & 3.13 & 3.02 & 3.27 & 3.29 & 3.18
\end{tabular}

\begin{tabular}{ccccc|ccccc}
\hline \multicolumn{8}{|c|}{ Sesiones de juego } \\
\hline \multirow{2}{*}{ Resumen } & RF & GP & MLP & SVM & RF & GP & MLP SVM \\
\hline Correlación & -0.29 & 0.25 & 0.11 & 0.40 & -0.16 & 0.01 & -0.09 & 0.08 \\
MAE & 17.12 & 9.24 & 15.18 & 6.23 & 13.90 & 11.44 & 14.49 & 10.39 \\
RMSE & 25.39 & 12.28 & 21.65 & 9.18 & 19.49 & 16.18 & 19.65 & 16.80 \\
\hline
\end{tabular}

al utilizar random forest y máquina de soporte vectorial como algoritmos de estimación para actividades de tipo videojuego de reto originaron un coeficiente de correlación superior al 0.70, los cuales son comparables a resultados obtenidos en experimentación orientada a adultos [16]. Así mismo, estos valores son más realistas, ya que se obtienen sin ninguna información previa de los jugadores.

\section{Conclusiones y trabajo futuro}

En este trabajo se presentó un método denominado EGEKN para la estimación de consumo de energía con base en el movimiento detectado por un sensor Kinect. Se usaron tres tipos de características para entrenar un modelo de regresión. A partir de la experimentación se observó que usando solo las características de movimiento los resultados no son muy buenos, sobre todo cuando se entrenan por separado modelos para videojuegos de reto y de acción. Los resultados mejoran cuando se incluye información sobre características propias del individuo, en particular mejoró en la evaluación de los videojuegos de reto. Cuando se usó la frecuencia cardíaca se obtuvieron mejores resultados. Usando MLP para el proceso de regresión y evaluando con 10FCV se obtuvo una correlación de 0.99 para todos los ejercicios, 0.95 para los videojuegos de reto y 0.92 para videojuegos de acción. Esto nos lleva a la conclusión, en primer lugar, de que las características contextuales, tanto información del usuario como otros sensores, aportan información valiosa para la estimación del consumo 
Estimación automática del gasto energético de la actividad física de niños en videojuegos...

Tabla 4. Resultados de la segunda experimentación utilizando solo características de trabajo mecánico, datos del usuario y actividad física realizada.

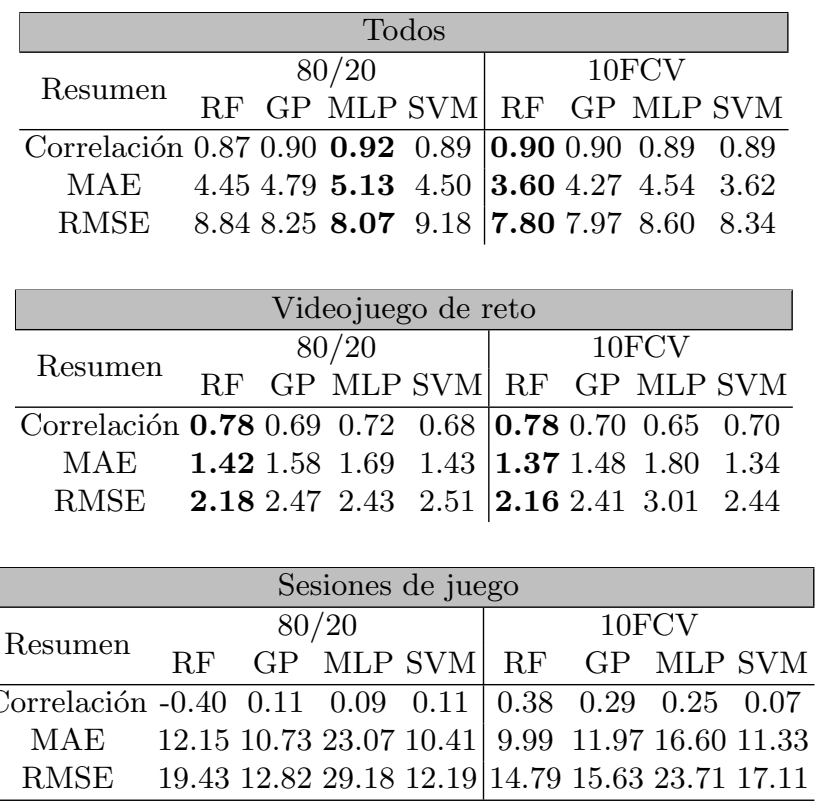

de energía. En segundo lugar, nos lleva a la conclusión de que es benéfico incluir en el entrenamiento una mayor variedad de movimientos y de que es más difícil estimar la energía consumida durante la ejecución de sesiones de videojuegos de acción que durante la ejecución de videojuegos de reto. Otra conclusión importante es que cada individuo tiene características propias de movimiento asociadas a su consumo energético, ya que cuando se evaluaron los modelos asegurando que no hubiera muestras de un mismo individuo en los conjuntos de entrenamiento y prueba, los resultados empeoraron, mostrando que los modelos entrenados inicialmente no eran independientes del usuario. Como trabajo futuro será interesante avanzar en la integración del método EGEKN en los videojuegos con el objetivo de mostrar en tiempo real el gasto energético a los jugadores. Además, un aspecto a mejorar será la precisión de los modelos independientes del jugador. Para lograr una mejoría significativa consideramos necesario incrementar el tamaño de la base de datos así como probar otras características de movimiento. Finalmente, se pretende aplicar el método de caracterización de movimientos a otras áreas de aplicación como reconocimiento de actividades.

Agradecimientos. Este trabajo de investigación se ha llevado a cabo en el contexto del programa "Cátedras CONACyT" financiado por el Consejo Nacional de Ciencia y Tecnología (CONACyT). 
Benjamín Gutiérrez-Serafín, Humberto Pérez-Espinosa, Ismael Edrein Espinosa-Curiel, et al.

Tabla 5. Resultados de la tercera experimentación utilizando todas las características propuestas

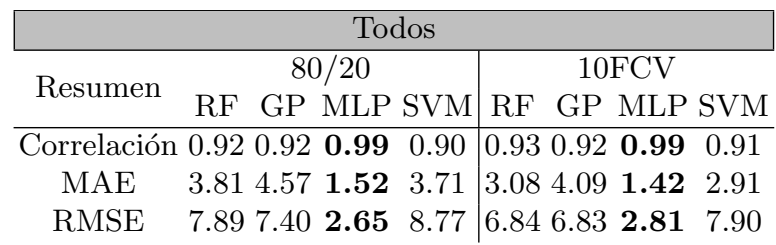

\begin{tabular}{|c|c|c|c|}
\hline \multicolumn{4}{|c|}{ Videojuego de reto } \\
\hline \multirow{2}{*}{ Resumen } & & $80 / 20$ & $10 \mathrm{FCV}$ \\
\hline & $\mathrm{RF}$ & GP MLP SVM & RF GP MLP SVM \\
\hline Correlación & 0.8 & $\begin{array}{lll}0.83 & \mathbf{0 . 9 3} & 0.82\end{array}$ & $\begin{array}{llll}0.85 & 0.84 & \mathbf{0 . 9 5} & 0.83\end{array}$ \\
\hline MAE & 1.24 & $\begin{array}{lll}1.26 & \mathbf{0 . 9 2} & 0.97\end{array}$ & $\begin{array}{llll}1.18 & 1.10 & \mathbf{0 . 6 8} & 0.80\end{array}$ \\
\hline RMSE & 1.85 & $\begin{array}{lll}1.92 & \mathbf{1 . 4 7} & 1.96\end{array}$ & $\begin{array}{llll}1.89 & 1.82 & \mathbf{1 . 0 3} & 1.89\end{array}$ \\
\hline
\end{tabular}

\begin{tabular}{cccc|ccccc}
\hline \multicolumn{8}{c|}{ Sesiones de juego } \\
\multirow{2}{*}{ Resumen } & \multicolumn{9}{c|}{$80 / 20$} & \multicolumn{4}{c|}{$10 \mathrm{FCV}$} \\
& RF & GP & MLP & SVM & RF & GP & MLP & SVM \\
\hline Correlación & 0.24 & 0.53 & $\mathbf{0 . 6 5}$ & 0.50 & 0.68 & 0.68 & $\mathbf{0 . 9 2}$ & 0.66 \\
MAE & 10.00 & 10.15 & $\mathbf{6 . 1 4}$ & 7.62 & 7.37 & 8.66 & $\mathbf{4 . 2 2}$ & 7.79 \\
RMSE & 15.24 & 12.38 & $\mathbf{9 . 4 0}$ & 10.89 & 11.93 & 11.70 & $\mathbf{6 . 3 0}$ & 12.34 \\
\hline
\end{tabular}

Tabla 6. Valores de correlación y errores concluyentes obtenidos al realizar experimentación con modelos entrenamiento y de prueba independientes.

\begin{tabular}{ccccc|cccc}
\hline \multicolumn{4}{c|}{$80 / 20$} \\
\multirow{2}{*}{ Resumen } & \multicolumn{4}{c|}{ Solo Videojuego de reto } & \multicolumn{4}{c|}{ Solo juegos } \\
& RF & GP & MLP & SVM & RF & GP & MLP SVM \\
\hline Correlación & 0.73 & 0.48 & 0.60 & $\mathbf{0 . 8 5}$ & 0.10 & 0.28 & 0.35 & 0.23 \\
MAE & 1.58 & 2.65 & 5.55 & $\mathbf{0 . 9 6}$ & 6.04 & 6.64 & 10.63 & 5.82 \\
RMSE & 2.42 & 2.92 & 6.14 & $\mathbf{1 . 3 8}$ & 7.99 & 8.67 & 16.97 & 7.22 \\
\hline
\end{tabular}

\section{Referencias}

1. Baranowski, T., Buday, R., Thompson, D., Lyons, E.J., Lu, A.S., Baranowski, J.: Developing Games for Health Behavior Change: Getting Started. Games For Health Journal 2(4), 183-190 (Aug 2013)

2. Cranz, A.: Microsoft kinect refuses to die (May 2018), https://gizmodo.com/microsoft-kinect-refuses-to-die-1825847023

3. De Leva, P.: Adjustments to zatsiorsky-seluyanov's segment inertia parameters. Journal of biomechanics 29(9), 1223-1230 (1996)

4. Dehghan, M., Akhtar-Danesh, N., Merchant, A.T.: Childhood obesity, prevalence and prevention. Nutrition Journal 4(1), 24 (Sep 2005)

5. Faigenbaum, A., Jr, J.E.M.: Guidelines for implementing a dynamic warm-up for physical education. Journal of Physical Education, Recreation \& Dance 78(3), 25$28(2007)$ 
6. Gardner, M.W., Dorling, S.: Artificial neural networks (the multilayer perceptron) - a review of applications in the atmospheric sciences. Atmospheric environment 32(14-15), 2627-2636 (1998)

7. Gelbart, M., Ziv-Baran, T., Williams, C.A., Yarom, Y., Dubnov-Raz, G.: Prediction of Maximal Heart Rate in Children and Adolescents. Clinical Journal of Sport Medicine: Official Journal of the Canadian Academy of Sport Medicine 27(2), 139-144 (2017)

8. Keytel, L., Goedecke, J., Noakes, T., Hiiloskorpi, H., Laukkanen, R., van der Merwe, L., Lambert, E.: Prediction of energy expenditure from heart rate monitoring during submaximal exercise. Journal of sports sciences 23(3), 289-297 (2005)

9. Kim, M., Angermann, J., Bebis, G., Folmer, E.: Vizical: Accurate energy expenditure prediction for playing exergames. In: Proceedings of the 26th annual ACM symposium on User interface software and technology. pp. 397-404. ACM (2013)

10. Knuttgen, H.G., Kraemer, W.J.: Terminology and measurement in exercise performance. The Journal of Strength \& Conditioning Research 1(1), 1-10 (1987)

11. Liaw, A., Wiener, M., et al.: Classification and regression by randomforest. R news $2(3), 18-22(2002)$

12. Liu, S., Gao, R.X., Freedson, P.S.: Computational methods for estimating energy expenditure in human physical activities. Medicine and Science in Sports and Exercise 44(11), 2138-2146 (Nov 2012)

13. Liu, Z., Tang, S., Qin, H., Bu, S.: Evaluating user's energy consumption using kinect based skeleton tracking. In: Proceedings of the 20th ACM international conference on Multimedia. pp. 1373-1374. ACM (2012)

14. Lu, A.S., Kharrazi, H., Gharghabi, F., Thompson, D.: A Systematic Review of Health Video Games on Childhood Obesity Prevention and Intervention. Games for health journal 2(3), 10.1089/g4h.2013.0025 (Jun 2013)

15. Mills, A., Rosenberg, M., Stratton, G., Carter, H.H., Spence, A.L., Pugh, C.J.A., Green, D.J., Naylor, L.H.: The effect of exergaming on vascular function in children. The Journal of Pediatrics 163(3), 806-810 (Sep 2013)

16. Nathan, D., Huynh, D.Q., Rubenson, J., Rosenberg, M.: Estimating physical activity energy expenditure with the kinect sensor in an exergaming environment. PloS one 10(5), e0127113 (2015)

17. Nishtar, S., Gluckman, P., Armstrong, T.: Ending childhood obesity: a time for action. The Lancet 387(10021), $825-827$ (2016)

18. Rasmussen, C.E.: Gaussian processes in machine learning. In: Summer School on Machine Learning. pp. 63-71. Springer (2003)

19. Smola, A.J., Schölkopf, B.: A tutorial on support vector regression. Statistics and computing 14(3), 199-222 (2004)

20. Soto, V., Calvo, M.G.: Parámetros inerciales para el modelado biomecánico del cuerpo humano. European Journal of Human Movement (2), 169-189 (1996)

21. Tsou, P.F., Wu, C.C.: Estimation of calories consumption for aerobics using kinect based skeleton tracking. In: 2015 IEEE International Conference on Systems, Man, and Cybernetics. pp. 1221-1226. IEEE (2015)

22. Willems, P., Cavagna, G., Heglund, N.: External, internal and total work in human locomotion. Journal of Experimental Biology 198(2), 379-393 (1995)

23. Williams, E.P., Mesidor, M., Winters, K., Dubbert, P.M., Wyatt, S.B.: Overweight and obesity: Prevalence, consequences, and causes of a growing public health problem. Current Obesity Reports 4(3), 363-370 (Sep 2015) 